\title{
Procesos de superficie en Harmonie-Arome y su importancia en procesos atmosféricos
}

\author{
Samuel Arístides Viana Jiménez ${ }^{1}$ (svianaj@aemet.es) \\ María Victoria Díez Muyo¹ (mdiezm@aemet.es)
}

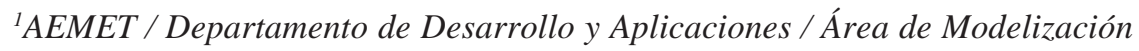

\begin{abstract}
RESUMEN
Los procesos superficiales representan un forzamiento de baja frecuencia para la atmósfera y son de gran importancia en modelización numérica del tiempo. En función del aumento de resolución de estos modelos, han ido incluyendo parametrizaciones más complejas y para un mayor número de procesos de superficie. Gran parte de ellas proceden del ámbito de los modelos climáticos, en los que se hace fundamental describir detalladamente las componentes no atmosféricas del sistema climático, en las que los procesos superficiales tienen lugar.
\end{abstract}

En este trabajo se describe brevemente el módulo SURFEX de modelización de superficie implementado en Harmonie-Arome, el modelo determinista operativo en AEMET. Posteriormente se describe el sistema de asimilación de superficie y su importancia en la corrección de las condiciones iniciales. También se presentan una serie de índices de humedad del suelo, fundamentales para describir el estado de la superficie del modelo, y se analiza su influencia en variables atmosféricas como la temperatura y humedad cerca del suelo o la amplitud térmica, así como en procesos atmosféricos como la convección. Por último, se describen otros esquemas avanzados de suelo y nieve disponibles en SURFEX, que se activarán en futuras versiones del modelo.

PALABRAS CLAVE: predicción numérica; superficie; asimilación de superficie; modelización; humedad del suelo.

\section{INTRODUCCIÓN}

AEMET, mediante su participación en el consorcio HIRLAM, colabora en el desarrollo del sistema de predicción numérica Harmonie-Arome. Se trata de un modelo numérico no hidrostático de área limitada y alta resolución (BENGTSSON et al., 2017). Harmonie-Arome se ejecuta diariamente en AEMET 8 veces al día, para dos dominios distintos (Península+Baleares y Canarias). Desde junio de 2017 constituye el modelo operativo de corto plazo de AEMET.

El desarrollo de Harmonie-Arome se organiza en torno a distintos grupos de trabajo en los que participa personal de los servicios meteorológicos de los distintos países miembros del consorcio: grupo de sistemas, dinámica, física, superficie, etc. Cada año se elabora un plan de trabajo (rolling work plan) en el que cada grupo detalla las actividades previstas para el desarrollo del modelo. Periódicamente, cuando se completa un número suficiente de estos desarrollos, se agrupan para configurar una nueva versión o ciclo del modelo. Tras un periodo de validación objetiva y verificaciones en distintos periodos y dominios, si los resultados son satisfactorios estos ciclos se «liberan» para su progresiva implantación operativa por parte de los distintos servicios meteorológicos miembros. Actualmente en AEMET se ejecuta operativamente el ciclo 40. Paralelamente, se trabaja en el desarrollo del ciclo 43 del modelo. 


\section{SURFEX: EL MODELO DE SUPERFICIE DE HARMONIE-AROME}

La función de un modelo de superficie, en el contexto de la predicción numérica del tiempo (PNT), es simular los intercambios superficiales con la atmósfera, que tienen lugar fundamentalmente como flujos de momento, humedad y calor. Estos flujos actuarán como condiciones de contorno para el modelo atmosférico propiamente dicho. En el modelo Harmonie-Arome los procesos de superficie están representados mediante el modelo SURFEX (MASSON et al., 2013), que se integra de forma acoplada (on-line) al modelo atmosférico. SURFEX (del francés surface externaliseé) nace en el seno de Meteo-France a partir de 2005, para separar la modelización de los procesos de superficie del código atmosférico de su modelo operativo, por lo que es susceptible de ser explotada en numerosas aplicaciones fuera del mundo de la PNT.

La resolución de SURFEX en Harmonie-Arome es similar a la de la componente atmosférica del modelo, es decir que comparten la discretización horizontal, y ambas componentes se «comunican» a través del nivel más bajo del modelo atmosférico. Al igual que en otros modelos de superficie, se utiliza el paradigma de «teselas» para dar cuenta de la heterogeneidad superficial. Esto es, cada punto de grid de superficie se compone de hasta 4 tipos de superficie o teselas (en SURFEX son mar, lago, superficie urbana y superficie natural o nature) en proporciones variables. Cada una de estas teselas evoluciona mediante sus propios esquemas de parametrización, que en cada punto de grid se integran por separado en cada paso de tiempo: finalmente se promedian ponderadamente los flujos de las teselas que componen cada punto de grid, para obtener los flujos agregados que se comunicarán a la componente atmosférica. Es importante señalar que en SURFEX no existen interacciones laterales directas entre puntos de grid contiguos; cualquier posible interacción se producirá de manera indirecta a través de la componente atmosférica.

\subsection{Modelización de la humedad y temperatura del suelo}

En su configuración actual, Harmonie-Arome tiene activado el esquema ISBA 3-L para la evolución de la temperatura y la humedad del suelo en la tesela nature. Este esquema discretiza el suelo en 3 capas que se intercambian calor y humedad según una aproximación force-restore (NOILHAN y PLANTON, 1989). La primera capa, de espesor $1 \mathrm{~cm}$ y en contacto directo con la atmósfera, permite simular mejor la temperatura superficial, que regulará el flujo de calor a la atmósfera. La segunda capa (root) representa aproximadamente el nivel de las raíces de las plantas y la tercera capa (deep layer) constituye un reservorio inferior de humedad. Estas dos capas alcanzan profundidades típicas de $1 \mathrm{~m}$ y 1,5-2 m respectivamente, en función del tipo de suelo predominante en cada punto de grid (son más profundas en zonas boscosas). El flujo superficial de humedad se compone de la evaporación directa desde el suelo y la transpiración de las plantas (en ambos casos se tiene en cuenta el agua almacenada en toda la capa root). También se incluye una aportación adicional por la evaporación desde la superficie vegetal de la precipitación depositada sobre la misma, que también se parametriza.

Se establecen ecuaciones de evolución para el contenido acuoso $w$ de cada una de las capas, con términos de entrada en el sistema (precipitación y fusión de nieve), de salida (evapotranspiración, escorrentía, drenaje profundo) y de intercambio entre capas mediante movimientos de drenaje y de difusión entre las mismas. Para obtener más detalles sobre el esquema ISBA 3-L, puede consultarse el documento científico de SURFEX (Le Moigne et al., 2018), disponible en la página web del proyecto (https://www.umr-cnrm.fr/surfex/).

\section{2. Índices descriptivos de la humedad del suelo}

La capacidad de un suelo determinado de contener y retener agua en su seno es función del tipo de suelo, más concretamente de su textura o porosidad. Por ejemplo, suelos más arenosos (grano grueso) tendrán menor capacidad de contener agua en su interior, al contrario que los suelos arcillosos (grano más fino). Según aumenta el contenido acuoso presente en un suelo determinado, se pasará por tres puntos característicos. El punto de marchitez o wilting point $\left(w_{\text {wilt }}\right)$ es la concentración de agua en el suelo por debajo de la 
cual las raíces de las plantas no son capaces de extraer humedad del suelo por lo que estas no pueden sobrevivir. Por encima de este punto, según se aumenta en contenido acuoso, el suelo alcanzará su capacidad de campo ofield capacity $\left(w_{f c}\right)$, que se corresponde con la máxima cantidad de agua que un suelo es capaz de retener contra la gravedad. A partir de ese punto, el suelo va produciendo infiltración hacia capas inferiores. Finalmente, si el suelo sigue cargándose de humedad llega al punto de saturación $\left(w_{\text {sat }}\right)$, a partir del cual ya no podrá admitir más agua, generándose escorrentía superficial. En SURFEX, los valores de estos puntos característicos en cada punto de grid dependen funcionalmente de los porcentajes de arcilla y arena, que se extraen de una base de datos fisiográfica. Actualmente en Harmonie-Arome se utilizan los mapas de la FAO (Food and Agriculture Organization). La figura 1 muestra los valores de estos puntos característicos sobre la península ibérica a partir de estos mapas.
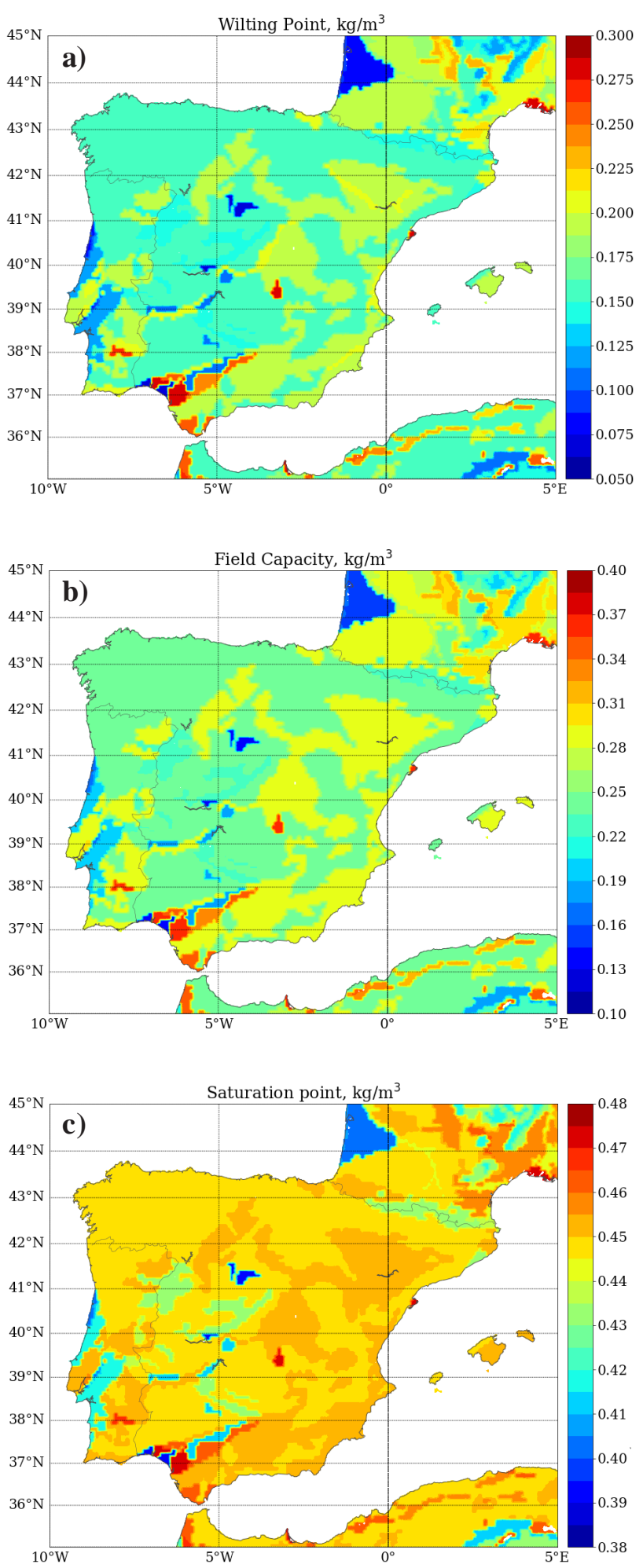

Los movimientos de agua entre capas por fuerzas de capilaridad o gravitatorias serán proporcionales a la diferencia entre el contenido acuoso de la capa y el correspondiente punto de marchitez o la capacidad de campo. Igualmente, el flujo de humedad hacia la atmósfera desde la capa root está influenciado por estos puntos característicos, al aumentar la eficiencia evaporativa linealmente entre el punto de marchitez y la capacidad de campo (figura 2). Por ello es interesante pasar a describir la humedad del suelo de forma relativa a estos puntos, pudiendo así comparar la capacidad evaporativa de los puntos de un dominio independientemente de su heterogeneidad en términos de contenido de arcilla y arena. Se definen así tres índices: la humedad residual (SMR), que mide el grado de sequedad del suelo, el índice de humedad del suelo (SWI), que mide la eficiencia de evaporación (toma valores negativos en suelos con estrés hídrico y superiores a la unidad por encima de la capacidad de campo), y el exceso de capacidad de campo (FCE), que mide el grado de saturación:

$$
\begin{aligned}
& S M R=w / w_{\text {wilt }} \\
& S W I=\left(w-w_{\text {wilt }}\right) /\left(w_{f c}-w_{\text {wilt }}\right) \\
& F C E=\left(w-w_{f c}\right) /\left(w_{\text {sat }}-w_{f c}\right)
\end{aligned}
$$

Estos índices evolucionan a lo largo del tiempo en respuesta a la intensidad de las fuentes y sumideros de humedad (precipitación, fusión de nieve, escorrentía, evapotranspiración, etc.). Así, resultan especialmente útiles para monitorizar el funcionamiento del modelo operativo y detectar sus sesgos sistemáticos.

Figura 1.

a) Punto de marchitez,

b) capacidad de campo, y

c) punto de saturación $\left(\mathrm{kg} / \mathrm{m}^{3}\right)$ en Harmonie-Arome. 


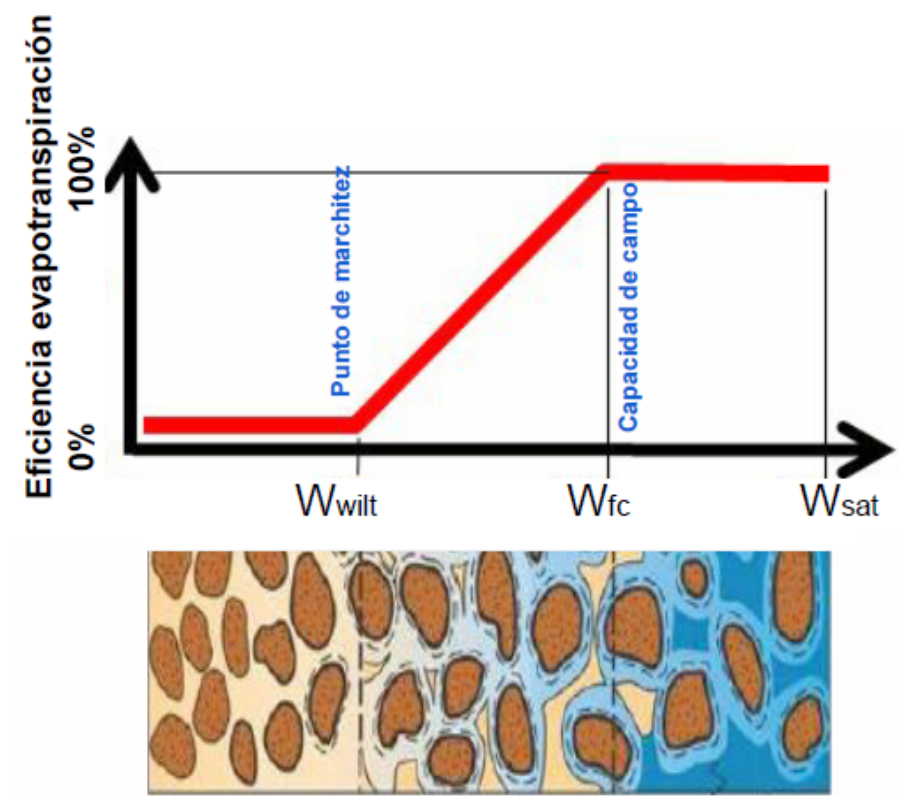

Figura 2.

Puntos característicos del suelo en relación a su contenido acuoso y evolución de la eficiencia evapotranspirativa.

Por ejemplo, un modelo operativo que produzca poca precipitación en invierno y primavera puede llevar a una superficie excesivamente seca en verano; esto a su vez puede afectar a la precipitación convectiva estival al generar un déficit de evaporación desde la superficie.

\section{LA ASIMILACIÓN DE SUPERFICIE EN HARMONIE-AROME}

La técnica de asimilación de superficie empleada en Harmonie-Arome sobre la tesela de naturaleza, es Interpolación Óptima, que fue descrita en MAHFOUF (1991), y empleada en otros modelos de PNT como el modelo Hirlam (RodRíGUEZ et. al., 2003).

La base de dicha técnica es usar las observaciones de temperatura y humedad a $2 \mathrm{~m}$ tomadas por las estaciones sinópticas para inicializar la temperatura y el contenido de agua en el suelo de la primera y la segunda capa. No es posible inicializar con esta técnica la temperatura y el contenido de agua del suelo de la tercera capa, pues no tiene una conexión «directa» con la atmósfera.

La técnica consta de dos partes bien diferenciadas. En primer lugar se realiza un análisis de la temperatura y humedad relativa a dos metros de las estaciones sinópticas, y posteriormente se «transfiere» dicha información al suelo.

\section{INTERACCIONES SUPERFICIE-ATMÓSFERA EN HARMONIE-AROME: APROXIMACIÓN ESTADÍSTICA}

El estudio de las interacciones superficie-atmósfera constituye un campo muy amplio de las ciencias de la tierra, al que se han dedicado múltiples esfuerzos en los últimos 20 años y en el cual, sin embargo, aún quedan muchas cuestiones por resolver. El número y la complejidad de los procesos físicos involucrados entre la superficie, la vegetación y la atmósfera, el amplio rango de escalas temporales en que estas interacciones tienen lugar, y la enorme heterogeneidad que presenta la superficie en un espectro igualmente amplio de escalas espaciales y temporales pueden citarse entre las razones que hacen que el conocimiento de las interacciones superficie-atmósfera continúe siendo relativamente limitado (SANTANELLO et al., 2017). No obstante, los avances producidos han permitido el desarrollo de gran variedad de modelos de superficie que describen procesos cada vez más complejos. 
El objetivo de esta sección es ilustrar el grado de conexión existente entre las condiciones locales de humedad del suelo y algunos procesos atmosféricos. Para ello haremos uso del archivo de la pasada operativa de Harmonie-Arome 40h1.1 en AEMET sobre el dominio peninsular, entre los meses de mayo a agosto de 2017 y 2018. Ambos años fueron marcadamente distintos en cuanto a precipitaciones, siendo el invierno y primavera previos marcadamente secos en 2017 y bastante húmedos en cuanto a precipitaciones en 2018, por lo que se espera observar diferencias significativas en cuanto a la reserva de humedad en el suelo del modelo. En el periodo elegido (final de primavera y época estival) se espera que los efectos directos de la superficie sobre la atmósfera sean mayores que en otras épocas del año, al minimizarse el efecto de los grandes sistemas sinópticos (frentes, borrascas...) y cobrar importancia los procesos de acoplamiento local entre la superficie continental y la atmósfera (convección y nubosidad de evolución diurna, tormentas de masa de aire, etc.).

\subsection{Diferencias en la humedad del suelo en los dos periodos}

En la figura 3 se puede comparar el estado medio de la humedad del suelo sobre la península ibérica en los dos periodos analizados, en base a los índices definidos en la sección 2.2. Pueden observarse efectivamente diferencias en las zonas más secas y en estrés hídrico (tonos terrosos), tanto en el grado de sequedad (los tonos son más oscuros en 2017) como en la extensión de las zonas secas. Igualmente, las áreas con capacidad evaporativa (tonos amarillos-verdes) son más extensas en 2018.
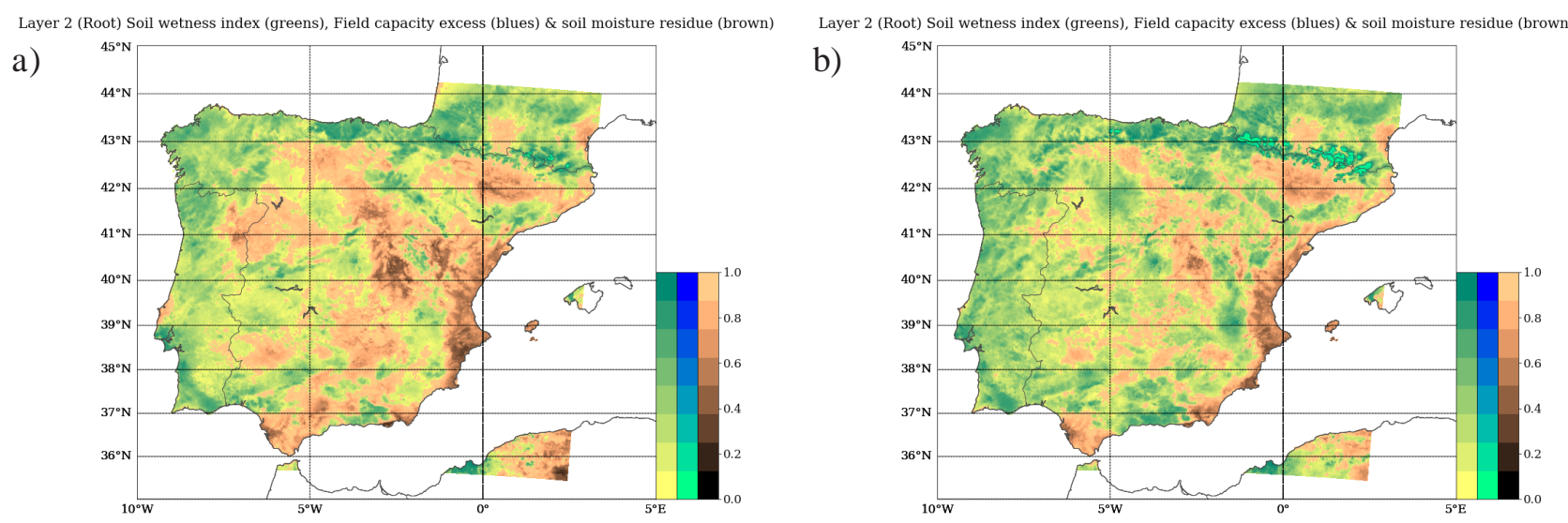

Figura 3. Índices medios de SMR, SWI y FCE sobre la Península en el periodo mayo-agosto de a) 2017 y b) 2018.

Puede obtenerse una evaluación más objetiva de estas diferencias mediante los histogramas del índice SWI (figura 4) para ambos periodos. En la capa 2 - root layer — se observa que la proporción de zonas secas $(S W I<0)$ es significativamente mayor en 2017 respecto de 2018. Igualmente, en la capa 3 se aprecia una mayor proporción de valores de $S W I>0,5$.
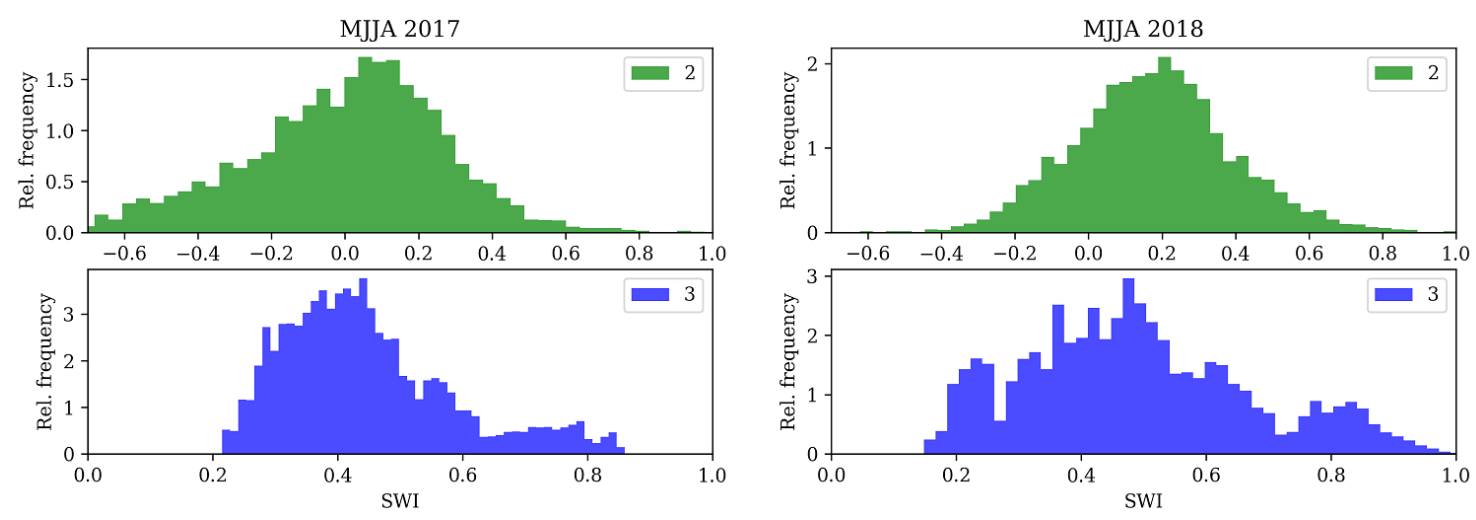

Figura 4. Histograma del índice de humedad del suelo en las capas 2 y 3 del modelo de mayo a agosto de 2017 y 2018. 


\subsection{Interacciones locales superficie-atmósfera}

A la hora de estudiar la sensibilidad de procesos atmosféricos sobre superficie natural a las condiciones locales de humedad del suelo, es conveniente hacer extensivo el concepto de «local» hasta un área superior a la rejilla del modelo, para ilustrar el hecho de que incluso bajo condiciones estacionarias muy influenciadas por la mesoescala, las circulaciones locales pueden desplazar horizontalmente las parcelas de aire más cercanas al suelo (receptoras de los flujos superficiales) por espacio de varios kilómetros en el transcurso de pocas horas. Igualmente, estas parcelas humedecidas desde la superficie, bajo condiciones de disparo convectivo, ascenderán hacia capas altas generando precipitación que podrá descargar a decenas de kilómetros. Por esta razón, los resultados presentados en esta sección se han obtenido a partir de salidas del modelo sometidas a un proceso de upscaling: la resolución de todos los campos se ha degradado a una rejilla de $45 \times 45 \mathrm{~km}$, promediando en cada punto los valores de todas las variables en la rejilla antigua. Además, para las estadísticas que se mostrarán a continuación se han filtrado los datos seleccionando únicamente puntos de la España peninsular con fracción de tierra superior a 1 sobre la resolución degradada. De esta forma quedan fuera gran parte de los puntos más cercanos a la costa.

En la figura 5a se puede apreciar el grado de control que ejerce la humedad del suelo en el flujo de calor latente superficial. Tal y como era esperable, en promedio los suelos más húmedos tienden a evaporar más agua hacia la atmósfera, si bien la relación no es unívoca y entran en juego múltiples factores adicionales considerados por el modelo de suelo (velocidad del viento, estabilidad térmica junto a la superficie, humedad relativa, etc.). Además, en el flujo de calor latente superficial también interviene, además de la evaporación directa del suelo, la evaporación y transpiración de la superficie vegetal, que es función de otras variables representadas en el esquema ISBA, como el índice de área foliar. Por todo ello la dispersión en los datos es significativa: para poder observar tendencias generales que faciliten la interpretación, se mostrarán los valores promediados por intervalos de la variable de abscisas (seleccionados de forma que contengan igual número de puntos). El grado de dispersión en los datos se presenta mediante barras de error con longitud igual a la desviación estándar de los puntos (figura 5b).
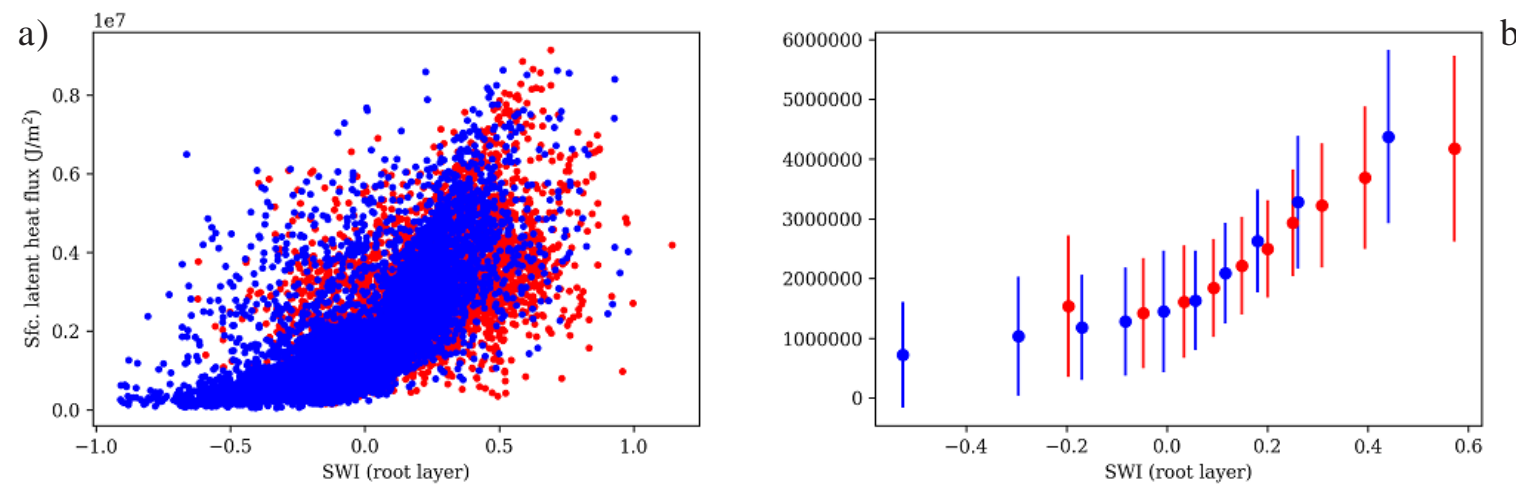

Figura 5. Sensibilidad del flujo de calor latente a las condiciones de humedad en el suelo.

En la figura 6 se muestra la evolución de la humedad relativa a $2 \mathrm{~m}$ y la diferencia de temperatura entre las 15 y las 06 UTC (como aproximación a la amplitud térmica diurna) en función de la partición entre los flujos superficiales de calor sensible y latente (LHF/SHF, también llamado ratio de Bowen inverso). Se observa un aumento gradual de la humedad relativa a $2 \mathrm{~m}$ a medida que la componente evaporativa gana peso en el flujo total de calor hacia la atmósfera (incremento en LHF/SHF). Al mismo tiempo, la amplitud térmica disminuye fuertemente. La distribución de valores en la gráfica, que muestra diferencias entre los dos periodos, permite hacer comparaciones de carácter climatológico entre los dos periodos (dado que el tamaño total de las series con las que se han calculado los promedios por intervalos es similar). Así, el periodo de 2018 estuvo caracterizado en promedio por amplitudes térmicas algo inferiores; de ahí se puede intuir que las temperaturas fueron algo más suaves en el periodo de 2018, como así sucedió (el verano de 2017 en la España peninsular fue muy cálido, mientras que el de 2018 fue cálido según los boletines climatológicos estacionales de AEMET). 
Por otra parte, si estudiamos la distribución de estas dos variables con el SWI (figura 7) puede observarse en primer lugar un aumento importante en la dispersión de los datos. Además, para valores bajos $(<0,2) \mathrm{o}$ negativos del $S W I$, no se observa influencia de la humedad del suelo sobre la humedad y amplitud térmica modelizadas a $2 \mathrm{~m}$. En cambio, los suelos en mayor disposición de evapotranspirar ( $S W I>>0,2)$ disminuyen la amplitud térmica y controlan la humedad relativa al menos en cierto grado (ya que la dispersión sigue siendo importante para este rango de humedades).
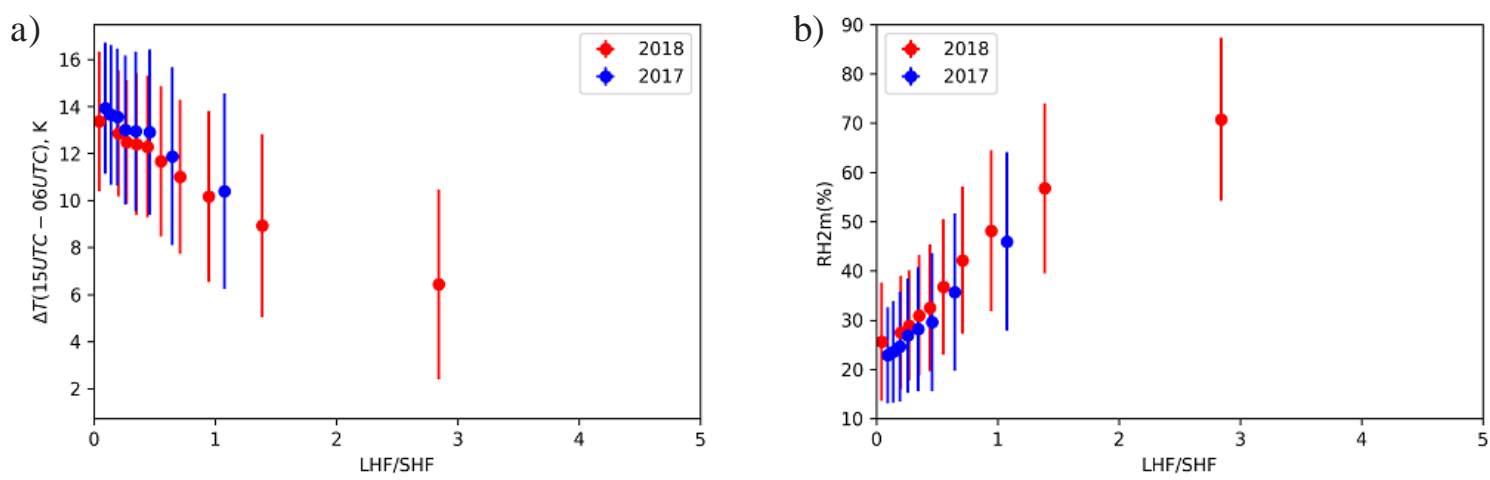

Figura 6. Influencia del ratio de calor sensible/latente sobre la amplitud térmica diurna y la humedad relativa a $2 \mathrm{~m}$.
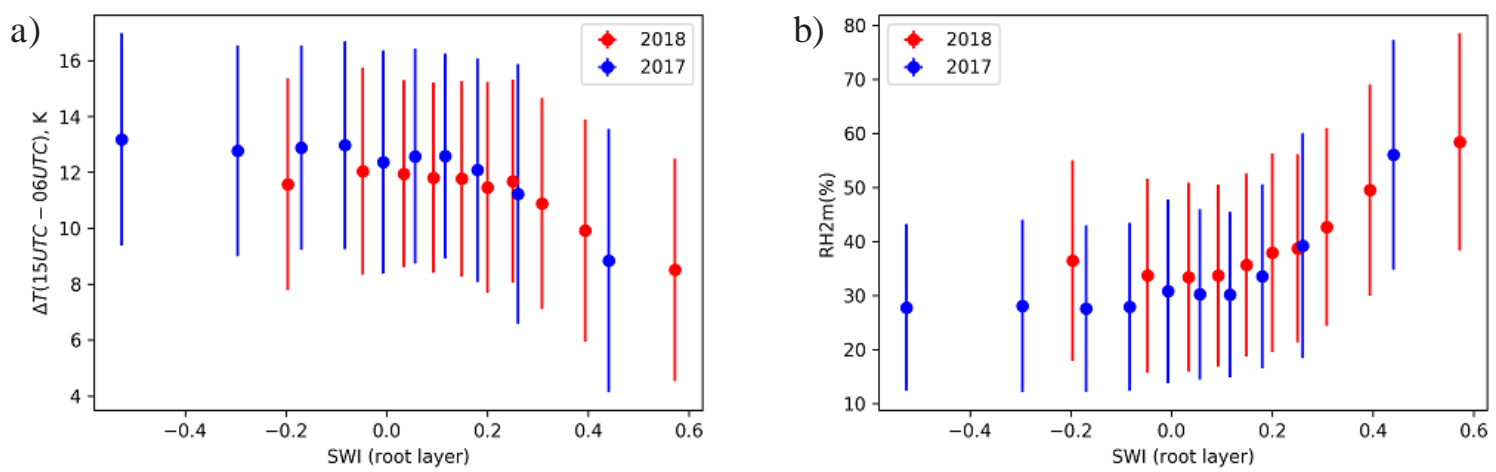

Figura 7. Influencia de la humedad del suelo (capa root) sobre la amplitud térmica diurna y la humedad relativa a $2 \mathrm{~m}$.

A continuación, estudiaremos la interacción superficie-atmósfera en conexión con la precipitación. En la formación de precipitación en torno a un área determinada intervienen un gran número de procesos atmosféricos y la evaporación local desde la superficie será solo uno de ellos. La precipitación estratiforme por ejemplo guardará poca relación con las condiciones locales. Por ello, las conexiones con las variables de superficie serán probablemente más difíciles de apreciar. No obstante, en el periodo analizado es de esperar que la precipitación vespertina (12-18 UTC) se encuentre frecuentemente asociada a la convección diurna forzada por el calentamiento de la superficie en las horas previas. Para restringir el estudio a este tipo de proceso, se ha optado por seleccionar del conjunto de datos únicamente aquellos con precipitación acumulada inferior a $1 \mathrm{~mm}$ y CAPE $<200 \mathrm{~J} / \mathrm{kg}$. Aunque el umbral de precipitación parezca bajo, hay que tener en cuenta que se refiere al grid degradado, por lo que a la resolución original se corresponderá con una precipitación mayor (en un área más reducida).

Analizando en primer lugar la relación entre los flujos superficiales de calor latente y sensible con las cantidades de precipitación «local», puede observarse (figura 8) una dependencia (inversa) clara con el flujo superficial de calor sensible (pendiente más pronunciada y menor dispersión). Para el flujo de calor latente, la dependencia (de signo opuesto) parece menos pronunciada y se limita a valores del flujo inferiores a $3 \cdot 10^{6} \mathrm{~J} / \mathrm{m}^{2}$ aproximadamente (por encima de ese valor, la pendiente de los puntos parece anularse o incluso invertir la tendencia para los flujos más altos). 

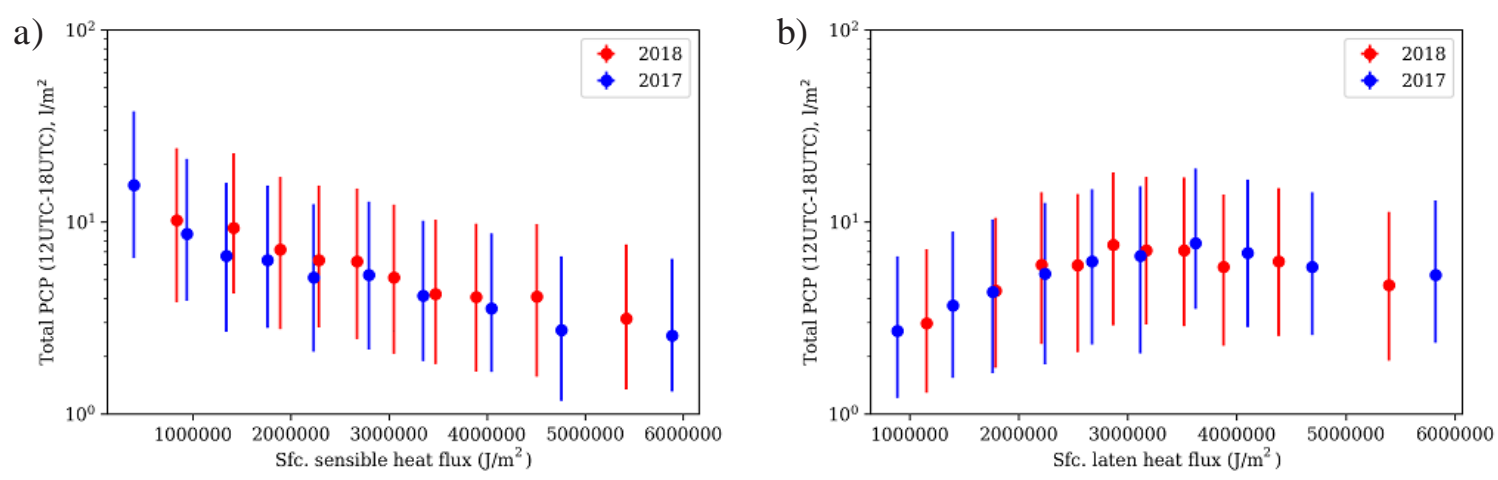

Figura 8. Influencia de los flujos superficiales de calor sensible y latente sobre las acumulaciones locales de precipitación entre las 12 y 18 UTC.

En cuanto a la influencia directa del contenido de humedad en el suelo sobre las cantidades de precipitación vespertinas (figura 9), no se aprecia influencia para $S W I<0,2$, al igual que se ha observado con la humedad relativa y la amplitud térmica. Por encima de ese valor se intuye un desplazamiento hacia precipitaciones más intensas para suelos más húmedos, aunque la dispersión es significativa. Así para la precipitación, aunque sí puede observarse una correlación con los flujos de superficie, no es posible establecer una interconexión clara con las condiciones locales medias de humedad del suelo. El degradado de resolución empleado puede serinsuficiente, en comparación con el desplazamiento típico de una célula convectiva terrestre, para desvelar la posible interconexión mediante este procesado estadístico sencillo. Igualmente, tal vez sea necesario un mayor filtrado de los datos (por ejemplo restringiendo la selección a puntos con mayor distancia a la costa o a días con convección de masa de aire). La complejidad de los mecanismos de interacción intermedios entre la humedad del suelo y la formación de precipitación convectiva hace necesarios enfoques más elaborados para su estudio, como el Heated Condensation Framework (HCF; TAWFIK y DIRMEYER, 2014), que diagnostica la contribución de los flujos de superficie al inicio de la convección en función de los perfiles atmosféricos de temperatura y humedad.

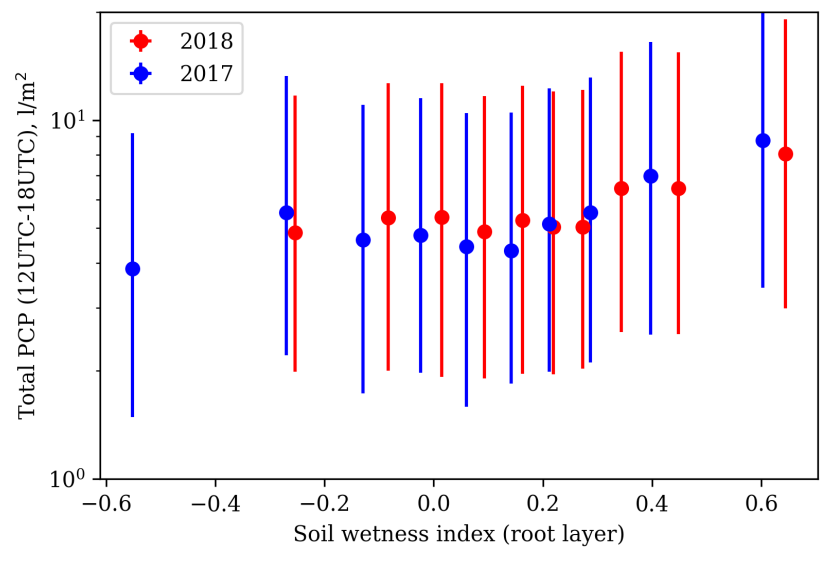

Figura 9. Influencia de la humedad del suelo (capa root) sobre las acumulaciones locales de precipitación entre las 12 y 18 UTC.

\section{FUTURAS OPCIONES DE SUPERFICIE EN HARMONIE-AROME}

Tradicionalmente, en el desarrollo de un modelo operativo de predicción se busca maximizar su rendimiento en la verificación de variables atmosféricas en las escalas propias de la PNT: por ello, la complejidad requerida en la representación de la superficie del modelo ha ido ligada a los objetivos de mejora en la representación atmosférica. Actualmente, las configuraciones operativas de Hamonie-Arome no incluyen algunas de las opciones de SURFEX más avanzadas para la representación de superficie, que se desarrollaron principalmente para su uso en modelos climáticos, simulación hidrológica, nivología, etc. A continuación se resumen algunas de las opciones avanzadas de superficie en cuya incorporación en los próximos ciclos del modelo se está trabajando actualmente (más detalles en Le Moigne et al., 2018):

- Soil diffusion scheme (DIF). Este esquema discretiza la columna de suelo en un mayor número de capas (habitualmente 12), distinguiendo igualmente un estrato ocupado por las raíces de las plantas y otro profundo. Las temperaturas se obtienen mediante el cálculo explícito de los flujos de calor entre 
capas mediante la ecuación de difusión (ley de Fourier), teniendo en cuenta la componente acuosa para el cálculo de la capacidad calorífica de cada capa. Por su parte, para obtener el contenido acuoso de las capas se resuelve la ecuación de Richards. Independientemente del aumento en la resolución vertical, en conjunto este esquema constituye una representación físicamente más consistente de los procesos térmicos e hidrodinámicos en el suelo.

- Explicit snow scheme (ES). Es un esquema multicapa (hasta 12 capas) que representa algunos de los procesos internos del manto nivoso: transmisión de radiación, congelación-descongelación, compactación y estabilización o el almacenamiento de agua líquida y su movimiento entre capas. Los intercambios energéticos y de masa con la atmósfera y el suelo quedan mejor representados.

- Balance energético múltiple (MEB). Esta opción reformula completamente el balance energético en la tesela nature, computando por separado balances para suelo desnudo, vegetación y manto nivoso, y considerando interacciones entre estas tres superficies hasta ahora no representadas, como por ejemplo entre la nieve y la vegetación parcialmente cubierta por ella. La resistencia aerodinámica se hace depender del reparto entre las tres superficies, lo que permite resolver mejor temperaturas y vientos junto al suelo.

- Opciones de hidrología subgrid (SGH). En los modelos numéricos la distribución de la precipitación entre escorrentía e infiltración es crucial a la hora de simular el balance hidrológico, lo que influye fuertemente en la componente atmosférica, especialmente en simulaciones climáticas. Las distintas versiones de suelo en ISBA consideran la escorrentía en sus ecuaciones dinámicas, sin embargo una parte importante de la misma ocurre a escalas inferiores a la resolución elegida, por lo que se hace necesario introducir parametrizaciones que den cuenta de estos procesos. Mediante la incorporación de estas opciones además de mejorar los forzamientos atmosféricos se pretende aumentar la utilidad de la superficie simulada en Harmonie-Arome en el ámbito de las aplicaciones hidrológicas.

Será necesario evaluar la calidad de estos nuevos esquemas de superficie en su ejecución acoplada con el modelo atmosférico. Para ello, se realizarán simulaciones largas corriendo el modelo en versión climática (sin asimilación), para determinar sus sesgos sistemáticos comparando las salidas frente a distintos conjuntos de observaciones (reanálisis, productos satelitales, etc.). Una vez eliminados o reducidos estos sesgos se introducirá la asimilación de datos, que se espera incluya algoritmos más avanzados de tipo variacional, adaptados a nuevos tipos de observaciones de teledetección (humedad del suelo, cobertura de nieve, etc.).

\section{CONCLUSIONES}

Los esquemas de superficie tratan de representar la heterogeneidad de los procesos superficiales con el objeto de proporcionar forzamientos de superficie adecuados para la parte atmosférica del modelo. Se han presentado las características generales del esquema de Harmonie-Arome para la superficie natural, que utiliza un modelo simplificado de 3 capas de suelo con ecuaciones de evolución de la temperatura y la humedad a partir de las que se determinan los flujos superficiales mediante ecuaciones de diagnóstico. Estos flujos son muy sensibles a los valores iniciales de temperatura y sobre todo humedad del suelo, por lo que la asimilación de superficie juega un papel importante.

La humedad del suelo muestra marcadas diferencias entre los dos periodos analizados, asociadas al distinto carácter climatológico de las precipitaciones durante los meses estudiados y en las estaciones previas. Las condiciones locales de humedad y temperatura atmosféricas muestran correlacionarse con el contenido de humedad de la capa root; esta correlación es consistente con la interacción esperable entre la humedad del suelo y las condiciones locales de la baja atmósfera en la época del año estudiada. Sin embargo la correlación con la precipitación es mucho más débil e incierta debido a los numerosos mecanismos intermedios implicados, aspectos relativos a la resolución utilizada, etc.

Por último, se han resumido las características básicas de los esquemas más sofisticados de suelo, nieve e hidrología que se incluirán en la superficie de las futuras versiones de Harmonie-Arome. 


\section{AGRADECIMIENTOS}

Este trabajo se ha realizado en el marco de la Red Temática WiPSiS, financiada por el proyecto CGL2016-81828-REDT/AEI del Gobierno de España.

\section{REFERENCIAS}

Bengtsson, L., Andrae, U., Aspelien, T., Batrak, Y., Calvo, J., de Rooy, W., Gleeson, E., Hansen-Sass, B., Homleid, M., Hortal, M., Ivarsson, K., Lenderink, G., Niemelä, S., Nielsen, K. P., Onvlee, J., Rontu, L., Samuelsson, P., Muñoz, D. S., Subías, A.,Tum, S., Toll, V., Yang, X. y Køltzow, M. Ø., 2017. The HARMONIE-AROME Model Configuration in the ALADIN-HIRLAM NWP System. Mon. Wea. Rev., 145, 1919-1935.

Mahfouf, J.-F., 1991. Analysis of Soil Moisture from Near-Surface Parameters: A Feasibility Study. Journal of Applied Meteorology, 30, 1534-1547.

Masson, V., Le Moigne, P., Martin, E., Faroux, S., Alias, A., Alkama, R., Belamari, S., Barbu, A., Boone, A., Bouyssel, F., Brousseau, P., Brun, E., Calvet, J.-C., Carrer, D., Decharme, B., Delire, C., Donier, S., Essaduini, K., Gibelin, A.-L., Giordani, H., Habets, F., Jidane, M., Kerdraon, G., Kourzeneva, E., Lafaysse, M., Lafont, S., Lebeaupin Brossier, C., Lemonsu, A., Mahfouf, J.-F., Marguinaud, P., Mokhtari, M., Morin, S., Pigeon, G., Salgado, R., Seity, Y., Taillefer, F., Tanguy, G., Tulet, P., Vincendon, B., Vionnet, V. y Voldoire, A., 2013. The SURFEXv7.2 land and ocean surface platform for coupled or offline simulation of earth surface variables and fluxes. Geosci. Model Dev., 6, 929-960.

Nollhan, J. y Planton, S., 1989: A Simple Parameterization of Land Surface Processes for Meteorological Models. Mon. Weather Rev., 117, 536-549.

Rodríguez, E., Navascués, B., Ayuso, J.-J. y Järvenoja, S., 2003. Analysis of surface variables and parametrization of surface processes in Hirlam. Part I: Approach and verification by parallel runs. Hirlam Technical Report, 58 pp.

Le MoIgne, P. (editor), 2018. Surfex scientific documentation. Disponible en: https://www.umr-cnrm.fr/surfex/.

Santanello, J. A., Dirmeyer, P. A., Ferguson, C. R., Findell, K. L., Tawfik, A. B., Berg, A., Ek, M., Gentine, P., Guillod, B. P., van Heerwaarden, C., Roundy, J. y Wulfmeyer, V., 2018. Land-Atmosphere Interactions: The LoCo Perspective. Bull. Amer. Meteor. Soc., 99, 1253-1272.

TAwfik, A. B. y Dirmeyer, P. A., 2014. A process-based framework for quantifying the atmospheric preconditioning of surface triggered convection. Geophys. Res. Lett., 41, 173-178. 